\title{
Realization of electron vortices with large orbital angular momentum using miniature holograms fabricated by electron beam lithography
}

E. Mafakheri, A. H. Tavabi, P.-H. Lu, R. Balboni, F. Venturi, C. Menozzi, G. C. Gazzadi, S. Frabboni, A. Sit, R. E. Dunin-Borkowski, E. Karimi, and V. Grillo

Citation: Appl. Phys. Lett. 110, 093113 (2017); doi: 10.1063/1.4977879

View online: http://dx.doi.org/10.1063/1.4977879

View Table of Contents: http://aip.scitation.org/toc/apl/110/9

Published by the American Institute of Physics

\section{Articles you may be interested in}

Highly efficient electron vortex beams generated by nanofabricated phase holograms

Applied Physics Letters 104, 043109 (2014); 10.1063/1.4863564

Photonic spin Hall effect by the spin-orbit interaction in a metasurface with elliptical nano-structures Applied Physics Letters 110, 101908 (2017); 10.1063/1.4978520

Reconfigurable conversions of reflection, transmission, and polarization states using active metasurface Applied Physics Letters 110, 121901 (2017); 10.1063/1.4979033

A radar-infrared bi-stealth structure based on metasurfaces

Applied Physics Letters 110, 063502 (2017); 10.1063/1.4975781

Controlling the optical spin Hall effect with light

Applied Physics Letters 110, 061108 (2017); 10.1063/1.4975681

Optofluidic Fano resonance photonic crystal refractometric sensors

Applied Physics Letters 110, 091105 (2017); 10.1063/1.4977563

\section{Scilight}

Sharp, quick summaries illuminating the latest physics research

\section{Sign up for FREE!}

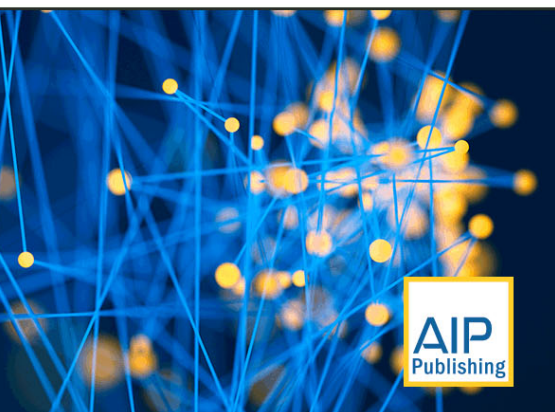




\title{
Realization of electron vortices with large orbital angular momentum using miniature holograms fabricated by electron beam lithography
}

\author{
E. Mafakheri, ${ }^{1}$ A. H. Tavabi, ${ }^{2}$ P.-H. Lu ${ }^{2}$ R. Balboni, ${ }^{3}$ F. Venturi, ${ }^{1,4}$ C. Menozzi,,${ }^{1,4}$ \\ G. C. Gazzadi, ${ }^{4}$ S. Frabboni, ${ }^{1,4}$ A. Sit, ${ }^{5}$ R. E. Dunin-Borkowski, ${ }^{2}$ E. Karimi, ${ }^{5,6}$ \\ and V. Grillo $4,7, a)$ \\ ${ }^{1}$ Dipartimento di Fisica Informatica e Matematica, Università di Modena e Reggio Emilia, via G Campi 213/a, \\ I-41125 Modena, Italy \\ ${ }^{2}$ Ernst Ruska-Centre for Microscopy and Spectroscopy with Electrons (ER-C) and Peter Grünberg Institute (PGI), \\ Forschungszentrum Jülich, D-52425 Jülich, Germany \\ ${ }^{3}$ CNR-IMM, Via P. Gobetti 101, I-40129 Bologna, Italy \\ ${ }^{4}$ CNR-Istituto Nanoscienze, Centro S3, Via G Campi 213/a, I-41125 Modena, Italy \\ ${ }^{5}$ Department of Physics, University of Ottawa, 25 Templeton, Ottawa, Ontario K1N 6N5 Canada \\ ${ }^{6}$ Department of Physics, Institute for Advanced Studies in Basic Sciences, 45137-66731 Zanjan, Iran \\ ${ }^{7}$ CNR-IMEM Parco Area delle Scienze 37/A, I-43124 Parma, Italy
}

(Received 27 December 2016; accepted 20 February 2017; published online 3 March 2017)

Free electron beams that carry high values of orbital angular momentum (OAM) possess large magnetic moments along the propagation direction. This makes them an ideal probe for measuring the electronic and magnetic properties of materials, as well as for fundamental experiments in magnetism. However, their generation requires the use of complex diffractive elements, which usually take the form of nano-fabricated holograms. Here, we show how the limitations of the current fabrication of such holograms can be overcome by using electron beam lithography. We demonstrate experimentally the realization of an electron vortex beam with the largest OAM value that has yet been reported to the first diffraction order $(\mathrm{L}=1000 \hbar)$, paving the way for even more demanding demonstrations and applications of electron beam shaping. Published by AIP Publishing.

[http://dx.doi.org/10.1063/1.4977879]

Similar to its optical counterpart, ${ }^{1}$ an electron vortex beam (EVB) possesses one or more phase singularities at the center of its helical wavefront and is an eigenstate of the component of orbital angular momentum (OAM) along its propagation direction with eigenvalue $\ell \hbar$ (where $\ell$ is an integer and $\hbar$ is the reduced Planck constant). ${ }^{2-5}$ As an electron is a charged particle, an EVB has a magnetic moment of $\ell \mu_{\mathrm{B}}$, where $\mu_{\mathrm{B}}$ is the Bohr magneton. Both its magnetic moment and its angular momentum allow for coupling to materials and for intriguing applications, including magnetic and shape dichroism measurements, ${ }^{6-9}$ chiral crystal structure characterization, ${ }^{10}$ nanoparticle manipulation, ${ }^{11}$ and electron spin polarization. ${ }^{12}$ EVBs are also of fundamental interest as they are characterized by a discrete quantum number that can form the basis of quantum experiments. ${ }^{13}$ For values of $\ell$ of a few units, the resulting magnetic effects are of the same order of magnitude as spin effects. However, the magnetic moment increases linearly with $\ell$ and can in principle be orders of magnitude larger, since there is no fundamental upper bound for $\ell$.

The realization of high OAM values is of great importance for the amplification of subtle physical effects. For example, a magnetic component of transition radiation has been predicted for large OAM beams. ${ }^{14}$ They have also been proposed for the measurement of out-of-plane magnetic fields in nanostructures using transmission electron microscopy (TEM) through the Larmor/Zeeman interaction. ${ }^{15,16}$

\footnotetext{
${ }^{\text {a) }}$ Author to whom correspondence should be addressed. Electronic mail: vincenzo.grillo@cnr.it
}

Moreover, large EVBs are interesting quantum objects in their own right. Whereas the TEM electron wavelength is typically on the order of $2 \mathrm{pm}$, a single highly twisted wavefront winds up with a step length of up to a few nanometers.

Finally, EVBs can be coupled to Landau states in the magnetic lens of a TEM (a longitudinal magnetic field). Landau states possess a functional similarity to the class of EVBs that are termed Laguerre-Gaussian beams and are characterized by a spiraling phase corresponding to $\mathrm{L}=\ell \hbar$ with a radial index $p . .^{13,17,18}$ The transverse energies of such states can be written as $\varepsilon=\hbar \Omega(2 p+\ell+|\ell|+1)$, where $\Omega=\mathrm{eB} / 2 \mathrm{~m}$ is the Larmor frequency, B is the magnetic field, and $m$ and $e$ are the electron mass and charge, respectively. Neglecting for the moment the large spread over the $p$ degree of freedom of most electron beams (e.g., in Ref. 19 we have shown an extreme case of dispersion in $p$ decomposition), for a typical magnetic field $\mathrm{B}$ of $2 \mathrm{~T}$ inside an electron microscope the discrete transverse energy of an excited state corresponding to a few $1000 \hbar$ can be as high as $0.5-1 \mathrm{eV}$ and can therefore potentially be coupled to infrared/visible light.

Unfortunately, the experimental realization of large OAM EVBs has been hindered technically by the approaches to nano-fabrication that have been used. Since EVBs were predicted theoretically, ${ }^{2}$ several different methods have been used to generate them, involving the use of spiral phase plates, $3,20-22$ pitch-fork holograms, ${ }^{4,5,19,23,24}$ spiral zone plates, ${ }^{25,26}$ Hilbert phase plates coupled to quadrupole lenses, ${ }^{27}$ multipole lenses in aberration correctors, ${ }^{28}$ and both magnetic ${ }^{29,30}$ and electrostatic $^{31}$ phase plates. From these options, off-axis phase holograms $5^{5,19,23,24,32}$ are still the method of choice. In such 
holograms, phase changes are introduced in proportion to their local thickness. EVBs have been reported with values for $\ell$ of $100-200 \hbar$ in high diffraction orders ${ }^{5}$ and a first-order value for $\ell$ of $200 \hbar$ with higher efficiency. ${ }^{19}$ In a very recent paper, one of the authors (V. Grillo), together with a group from the University of Oregon, demonstrated a superimposed set of vortices reaching up to $\mathrm{L}=4000 \hbar$ in the 5 th order. ${ }^{33}$ This beam is potentially useful in coupling with Landau states to reach $1 \mathrm{eV}$ energy but not in magnetic experiments where well-separated EVBs are necessary. ${ }^{15,16}$

Such phase holograms can be fabricated with different groove profiles to produce different intensity distributions in their diffraction orders. ${ }^{23}$ For example, a blazed triangular profile can potentially be used to convey all of the transmitted intensity into a single diffracted beam. ${ }^{23,34}$ Other less challenging and more popular groove profiles take sinusoidal and rectangular forms. For a perfectly tuned thickness, a rectangular shape is superior to a sinusoidal shape, as it inhibits all even diffraction orders, including the 0th order.

The limited resolution of focused ion beam (FIB) milling, which has been used to fabricate the holograms in these examples, prevents the realization of higher OAM beams since the primary grating periodicity $P$ must be decreased to achieve an increasing OAM in order to avoid the superposition of electron vortices of different diffraction orders. For definiteness, a top-hat cutoff of the hologram generates an EVB with size $r_{K} \approx \ell / R$, where $R$ is the radius of the cutoff in the hologram. The first diffraction order is centered at the main frequency $1 / P$ of the hologram. Therefore, the first order is only well sampled if $P \ll \frac{R}{2 \ell}$. Since $\mathrm{R}$ is typically limited to $\sim 100 \mu \mathrm{m}$, high spatial resolution is needed in the fabrication of large vortices. Limited resolution can also mean that an intended rectangular groove profile can end up being nearly sinusoidal. Moreover, it is demanding to maintain the same groove profile and a uniform response over both high and low spatial frequencies. Additional problems include the total patterning time and the total number of addressable pixels.

Here, we overcome these limitations by using electron beam lithography (EBL) to achieve a vortex with a topological charge as high as $1000 \hbar$. EBL is a technique that is used widely to produce patterns based on the selective electron irradiation of an electron sensitive material. We use a Zeiss $\Sigma$ scanning electron microscope equipped with a Schottky field emitter and a Raith Elphy Quantum pattern generator.

In order to optimize the spatial resolution of EBL patterning, we tested both positive and negative resists. We used square 50-nm-thick SiN membranes, on which the electron-transparent region had a width of $80 \mu \mathrm{m}$. The membranes were covered with evaporated Au (typically $200 \mathrm{~nm}$ thick), which was removed only in the hologram region. The procedure required 2 steps of lithography. A first step was used to create an electron transparent aperture in the $\mathrm{Au}$ mask in the active region of the hologram. A second step involved patterning the holograms with appropriate phase modulations. The pattern thickness was defined according to the formula (see supplementary material)

$$
t=\frac{1}{2} t_{0}\left(1+\operatorname{sign}\left(\sin \left(\ell \theta+\rho k_{\text {carrier }} \cos (\theta)\right)\right),\right.
$$

where $\rho, \theta$ are polar co-ordinates in the hologram plane, $k_{\text {carrier }}=2 \pi / P$ is the carrier frequency in the off-axis hologram, and sign[.] is the sign function, which is \pm 1 for positive and negative arguments, respectively. The thickness $t_{0}$ was chosen to provide a phase difference close to $\pi$. The rectangular groove shape defined by Eq. (1) conveniently allowed the use of EBL to produce holograms with 2 discrete thickness levels.

To first order, the separation $d$ between two hologram lines is related to the argument of the $\sin$ function, i.e., $f=\ell \theta+\rho k_{\text {carrier }} \cos (\theta)$, through the expression to the first order $\frac{1}{d} \approx \frac{\left|\nabla_{\rho, \theta}(f)\right|}{\pi}+\cdots$. When the function $f$ is stationary (i.e., when $\nabla_{\rho, \theta}(f)=0$ ), the separation between the lines increases. This condition is realized when $\rho=\ell / k_{\text {carrier }}, \theta=\frac{\pi}{2}$. A detailed analysis shows that the $f$-stationary point is a saddle point for $f$. Such a saddle point is present in our previous work for $\ell=200$, taking the form of a cross close to the center of the hologram. ${ }^{19}$ The lines in the hologram have a much higher frequency in the center and opposite the $f$-stationary point. As in our previous paper, we decided here to exclude the central region of the hologram from patterning. ${ }^{5,19}$ For all patterning, a bitmap image was created using the STEM_CELL software $^{35}$ and converted to a data format that was readable using the EBL pattern generator.

We made a first series of holograms by using polymethyl methacrylate (PMMA) for patterning and reactive ion etching (RIE) to transfer the pattern onto the SiN membrane (see supplementary material). This process was not able to provide a high enough carrier frequency. In contrast, Figure 1 shows the process that gave the best results in terms of resolution. In this case, we used hydrogen silsesquioxane (HSQ), which transformed into a silica-like structure after baking on a hot plate.
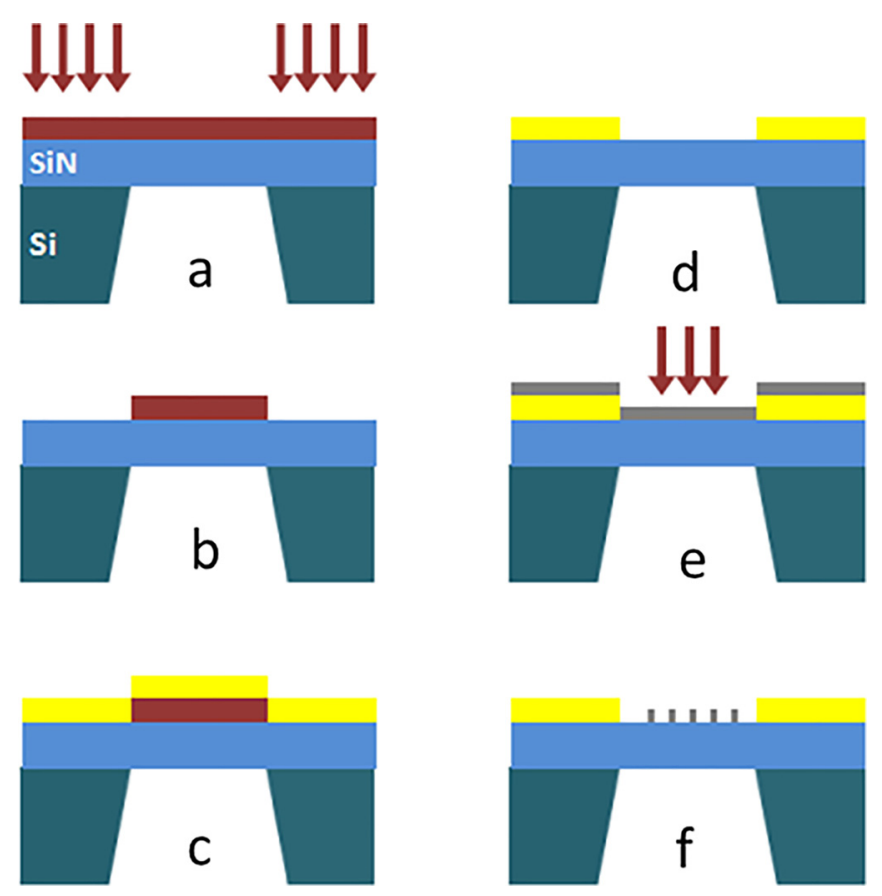

FIG. 1. Schematic diagrams showing (1) spin coating of PMMA and EBL; (2) developing the resist; (3) Au evaporation; (4) lift-off; (5) hydrogen silsesquioxane (HSQ) spin coating and EBL; and (6) developing the HSQ. 
The EBL was carried out at an accelerating voltage of $20 \mathrm{kV}$ using a $7.5 \mu \mathrm{m}$ aperture, an exposure dose of $700 \mathrm{pC} /$ $\mathrm{cm}$, and a beam current of $11 \mathrm{nA}$. After exposure, the sample was developed in tetramethylammonium hydroxide (TMAH) $2 \%$ for $90 \mathrm{~s}$ and rinsed in distilled water for $30 \mathrm{~s}$ followed by nitrogen drying.

Baked HSQ, which is resistant to electron beam irradiation, was the material used to impart a phase difference to the electron wave in the TEM. The use of such a negative resist provided considerable advantages in terms of ease of fabrication and well known superior resolution. The only disadvantage was the insulating nature of the HSQ, which resulted in charging during TEM examination. Each hologram was therefore coated with a few $\mathrm{nm}$ of evaporated $\mathrm{Cr}$, which solved most of the charging problems. The result of this patterning is shown in the form of a TEM image of a hologram in Figure 2(a). The patterned area is clearly visible, as is the large central hole. On the top-left, a region of stationary phase is visible (indicated by a circle). Although the image appears to show other stationary points, these are artifacts resulting from digital reproduction due to undersampling of the TEM image (further TEM and SEM images are also visible in the supplementary material). Figure 2(b) shows a TEM image of part of the hologram, which confirms that different spatial frequencies are reproduced correctly. Figure 2(c) shows a thickness map, calculated using energyfiltered TEM, ${ }^{19}$ of part of a pattern, in which line widths of $35 \mathrm{~nm}$ are present and the average periodicity is below

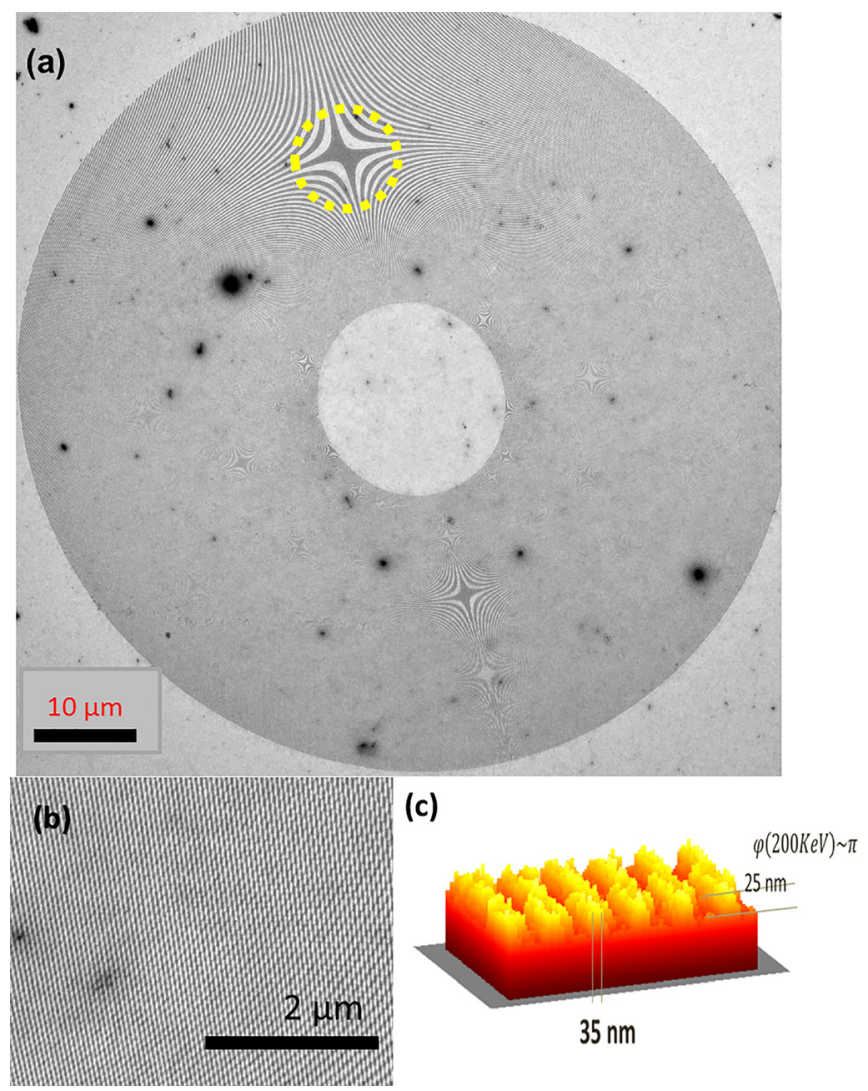

FIG. 2. (a) TEM image of an HSQ-based hologram for $\ell=1000 \hbar$. (b) Higher magnification TEM image of a region of the hologram, showing both low and high spatial frequencies in the pattern. (c) 3D rendering of an energy-filtered-TEM-based thickness map of a region of one of the holograms, showing detail on the order of $35 \mathrm{~nm}$.
$65 \mathrm{~nm}$, The map and simulations in the supplementary material indicate that, even at such a scale, the lateral definition of the trench is good, that the vertical step is nearly perfect, and that the trench thickness is uniform.

Figure 3(a) shows a nearly-in-focus image of a Fraunhofer diffraction pattern of the hologram recorded at $300 \mathrm{keV}$ using an FEI Titan equipped with a Schottky FEG and operated in LowMag mode. The two opposite vortices take the form of rings with uniformly bright intensities, confirming the very high resolution of our EBL pattern, i.e., that both high and low spatial frequencies are diffracted with the same intensity. We also observe only a very faint trace of second order diffraction, providing confirmation that the grooves are sharp and almost rectangular (as suggested by the thickness map). We achieved a first order efficiency of up to $20 \%$ but a second order efficiency of only $3.5 \%$. This inhibition of the second order is very important for applications and is a consequence of the highly rectangular profile of the gratings (see supplementary material).

We now address measurement of the OAM value of the vortex. In a previous article, ${ }^{19}$ the authors measured a thickness map in the hologram plane. Here, considering the large size of the pattern with respect to those fabricated previously, ${ }^{23}$ we are not able to make a reasonable thickness map of the entire hologram (we would need a digital image with a size of at least a $4 \times 10^{8}$ pixels). Therefore, we cannot check the exact OAM spectrum of the vortex in this way. Instead, inspired by a suggestion in the literature, ${ }^{36-38}$ we used the selected area diffraction (SAD) aperture to block half of the vortex. We then systematically varied the excitation of the diffraction lens, which is located immediately after the aperture. In this way, we observed the rotation of the Fresnel diffraction image. A simplified way to calculate this rotation angle makes use of the expression

$$
\theta=\left(\frac{\mathrm{eB}}{2 m} \pm \frac{\mathrm{L}}{m r^{2}}\right) \frac{\Delta z}{v},
$$

where $\Delta z$ is the propagation distance, $v$ is the electron velocity, and the quantity $r$ is a "semi-classical" value of the radius, whose correct quantum interpretation depends on the shape of the beam. ${ }^{38}$ The expression for the rotation angle contains two terms. The first is the Larmor rotation, which depends on the magnetic field of the diffraction lens. The second is referred to as the Gouy rotation and is associated with the phase gradient of the vortex. As the Gouy rotation goes to zero at large distances $r$ from the rotation center, we can separate the Larmor contribution by checking the rotation of the shadow of the aperture far from the center. For our large OAM vortex, as a result of the use of a weak diffraction lens, this contribution is small $(10 \%$ of the overall rotation), while the dominant contribution to the rotation originates from the phase gradient of the vortex itself.

For an exact evaluation of the Gouy rotation, instead of using Eq. (2) we evaluated the Fresnel propagation numerically for different values of $\ell$. We calibrated the defoci by comparing simulations and experimental images recorded without an aperture, since the absolute $z$ positions of both the SAD aperture and the Fraunhofer diffraction pattern were unknown. We define the parameter 


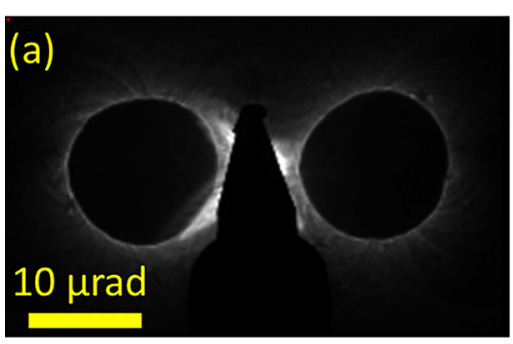

(c)

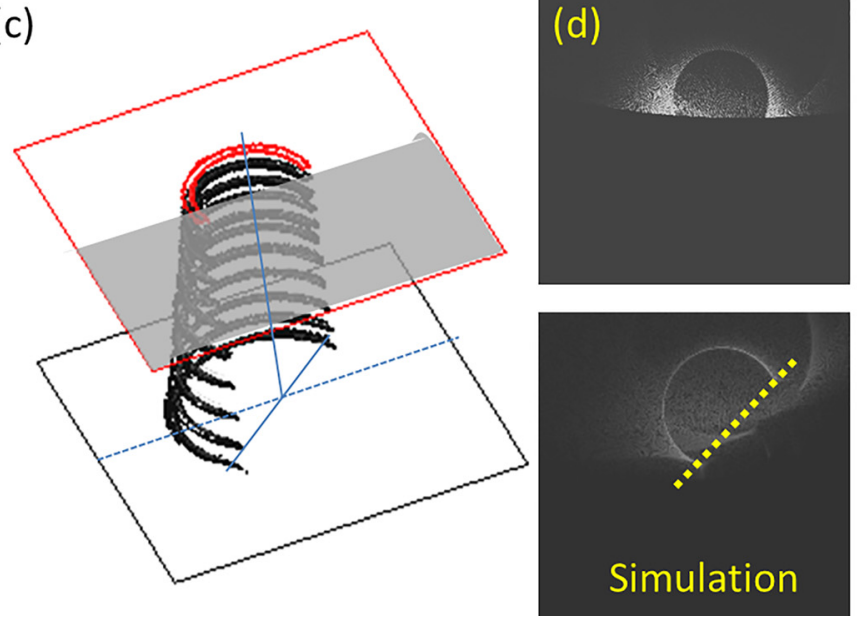

$$
z_{R}=\pi \frac{r_{\text {rim }}{ }^{2}}{1000 \times \lambda},
$$

which would be the Rayleigh range for an ideal LaguerreGauss beam with $p=0$ and an equivalent apparent rim radius $r_{r i m}$ (with the radius corresponding to maximal intensity). We find that the aperture is located at $z / z_{R}=0.5$ and that we analyzed the rotation after $z / z_{R}=2$. Figure $3(b)$ shows the expected rotation according to simulations for beams with different values of $L$ and for a realistic hologram structure. The best match for $\langle L\rangle$ is $(960 \pm 120) \hbar$ and is consistent with the nominal value. Figure 3(c) shows a comparison between a simulation for $L=1000 \hbar$ and our experimental results, demonstrating good agreement in the rotation angle (Minor details depend on the exact shape of the aperture).

To conclude, we have demonstrated that by using EBL we can overcome previous intrinsic limitations of holograms, in terms of (1) the maximum OAM that can be reached; (2) the minimum detail that can be reproduced (at least $35 \mathrm{~nm}$; in the supplementary material we reach $18 \mathrm{~nm}$ ); (3) improved uniformity of the frequency response; and (4) better suppression of higher order diffraction due to a nearly perfect rectangular groove profile. We believe that EBL will be the fabrication technique of choice for future complex diffractive optics with electrons. One of the most interesting perspectives is to use holograms to shape beams that have very well defined and stable properties, in order to increase the precision of TEM measurements. A very extended and precise grating can be used for electron interferometry, while by using an extended version of a conical hologram $^{32}$ it will be possible to create a nearly ideal Bessel beam potentially useful for interferometry or for measurements of small deflections.
FIG. 3. (a) Experimental image of a diffraction pattern of the hologram nearly in focus. A beam stop was used to block the transmitted beam. (b) Plot of the expected rotation as a function of OAM quantum number, shown together with our experimental measurement. (c) Schematic diagram illustrating the use of a knife edge to measure beam rotation. The half circles are the silhouette of the EVB after the knife edge (here in gray). (d) Experimental results and simulations for $\ell=1000$ before (upper figures) and after (lower figures) propagation. The simulations (left) and the experiment (right) are compared. The important parameter is the rotation, which is highlighted by the dashed line.

See supplementary material for: (S1) how to calculate the hologram, (S2) alternative EBL, (S3) imaging of the hologram, and (S4) discussions on efficiency vs groove shape.

V.G. acknowledges the support of the Alexander von Humboldt Foundation. We thank P. Pingue and F. Carillo for the access to the lithography facility of CNR-NEST in Pisa (Italy). S.F. and F.V. thank the University of Modena and Reggio Emilia for the grant FAR-2015-Project Title: Computer Generated Holograms for the realization and analysis of structured electron waves. The research leading to these results has received funding from the European Research Council under the European Union's Seventh Framework Programme (FP7/2007-2013)/ERC Grant Agreement No. 320832. E.K. and A.S. acknowledge the support of the Canada Research Chairs (CRC) and Canada Foundation for Innovations (CFI) Programs.

${ }^{1}$ D. L. Andrews and M. Babiker, The Angular Momentum of Light (Cambridge University Press, 2012).

${ }^{2}$ K. Y. Bliokh, Y. P. Bliokh, S. Savel'ev, and F. Nori, Phys. Rev. Lett. 99, 190404 (2007).

${ }^{3} \mathrm{M}$. Uchida and A. Tonomura, Nature 464, 737 (2010).

${ }^{4}$ J. Verbeeck, H. Tian, and P. Schattschneider, Nature 467, 301 (2010).

${ }^{5}$ B. J. McMorran, A. Agrawal, I. M. Anderson, A. A. Herzing, H. J. Lezec, J. J. McClelland, and J. Unguris, Science 331, 192 (2011).

${ }^{6} \mathrm{P}$. Schattschneider, S. Löffler, M. Stöger-Pollach, and J. Verbeeck, Ultramicroscopy 136, 81-85 (2014).

${ }^{7}$ S. Lloyd, S. M. Babiker, and J. Yuan, Phys. Rev. Lett. 108, 074802 (2012).

${ }^{8}$ A. Garcia and F. J. García de Abajo, Phys. Rev. Lett. 113, 066102 (2014).

${ }^{9}$ T. R. Harvey, J. S. Pierce, J. J. Chess, and B. J. McMorran, preprint arXiv:1507.01810.

${ }^{10}$ R. Juchtmans, A. Béché, A. Abakumov, M. Batuk, and J. Verbeeck, Phys. Rev. B 91, 094112 (2015).

${ }^{11}$ J. Verbeeck, H. Tian, and G. Van Tendeloo, Adv. Mater. 25, 1114-1117 (2013). 
${ }^{12}$ E. Karimi, L. Marucci, V. Grillo, and E. Santamato, Phys. Rev. Lett. 108, 044801 (2012).

${ }^{13}$ J. Harris, V. Grillo, E. Mafakheri, G. C. Gazzadi, S. Frabboni, R. W. Boyd, and E. Karimi, Nat. Phys. 11, 629 (2015).

${ }^{14}$ I. P. Ivanov and D. V. Karlovets, Phys. Rev. A 88, 043840 (2013).

${ }^{15}$ V. Grillo, T. R. Harvey, J. S. Pierce, F. Venturi, R. Balboni, G. C. Gazzadi, S. Frabboni, B. J. McMorran, R. W. Boyd, and E. Karimi, in Proceedings of the EMC 2016 (2016).

${ }^{16}$ A. Edström, A. Lubk, and J. Rusz, Phys. Rev. Lett. 116, 127203 (2016).

${ }^{17}$ P. Schattschneider, T. Schachinger, M. Stöger-Pollach, S. Löffler, A. Steiger-Thirsfeld, K. Y. Bliokh, and F. Nori, Nat. Commun. 5, 4586 (2014).

${ }^{18}$ E. Karimi, R. W. Boyd, P. de la Hoz, H. de Guise, J. Řeháček, Z. Hradil, A. Aiello, G. Leuchs, and L. L. Sánchez-Soto, Phys. Rev. A 89, 063813 (2014).

${ }^{19}$ V. Grillo, G. C. Gazzadi, E. Mafakheri, S. Frabboni, E. Karimi, and R. W. Boyd, Phys. Rev. Lett. 114, 034801 (2015).

${ }^{20}$ R. Shiloh, Y. Lereah, Y. Lilach, and A. Arie, Ultramicroscopy 144, 26 (2014).

${ }^{21}$ A. Béché, R. Winkler, H. Plank, F. Hofer, and J. Verbeeck, Micron 80, 16 (2014).

${ }^{22}$ V. Grillo, A. H. Tavabi, F. Venturi, H. Larocque, R. Balboni, G. C. Gazzadi, S. Frabboni, P.-H. Lu, E. Mafakheri, F. Bouchard, R. E. DuninBorkowski, R. W. Boyd, M. P. J. Lavery, M. J. Padgett, and E. Karimi, e-print arXiv:1609.09129v1.

${ }^{23}$ V. Grillo, G. C. Gazzadi, E. Karimi, E. Mafakheri, R. W. Boyd, and S. Frabboni, Appl. Phys. Lett. 104, 043109 (2014).

${ }^{24}$ T. R. Harvey, J. S. Pierce, A. K. Agrawal, P. Ercius, M. Linck, and B. J. McMorran, New J. Phys. 16, 093039 (2014).
${ }^{25}$ J. Verbeeck, H. Tian, and A. Béché, Ultramicroscopy 113, 83 (2012).

${ }^{26}$ K. Saitoh, Y. Hasegawa, N. Tanaka, and M. Uchida, J. Electron Microsc. 61, 171 (2012).

${ }^{27}$ P. Schattschneider, M. Stoger-Pollach, and J. Verbeeck, Phys. Rev. Lett. 109, 1-5 (2012).

${ }^{28}$ L. Clark, A. Béché, G. Guzzinati, A. Lubk, M. Mazilu, R. Van Boxem, and J. Verbeeck, Phys. Rev. Lett. 111, 064801 (2013).

${ }^{29}$ A. Béché, R. Van Boxem, G. Van Tendeloo, and J. Verbeeck, Nat. Phys. 10, 26-29 (2014).

${ }^{30}$ A. M. Blackburn and J. C. Loudon, Ultramicroscopy 136, 127 (2014).

${ }^{31}$ G. Pozzi, P.-H. Lu, A. H. Tavabi, M. Duchamp, and R. E. DuninBorkowski, "Generation of electron vortex beams using line charges via the electrostatic Aharonov-Bohm effect" (unpublished).

${ }^{32}$ V. Grillo, E. Karimi, G. C. Gazzadi, S. Frabboni, M. R. Dennis, and R. W. Boyd, Phys. Rev. X 4, 011013 (2014).

${ }^{33}$ B. J. McMorran, A. Agrawal, P. A. Ercius, V. Grillo, A. A. Herzing, T. R. Harvey, M. Linck, and J. S. Pierce, Phil. Trans. R. Soc., A 375, 20150434 (2017).

${ }^{34}$ V. Grillo, J. Harris, G. C. Gazzadi, R. Balboni, E. Mafakheri, M. R. Dennis, S. Frabboni, R. W. Boyd, and E. Karimi, Ultramicroscopy 166, 48 (2016).

${ }^{35}$ V. Grillo and E. Rotunno, Ultramicroscopy 125, 112 (2013).

${ }^{36}$ G. Guzzinati, P. Schattschneider, K. Y. Bliokh, F. Nori, and J. Verbeeck, Phys. Rev. Lett. 110, 093601 (2013).

${ }^{37}$ G. Guzzinati, L. Clark, A. Béché, and J. Verbeeck, Phys. Rev. A 89, 025803 (2014).

${ }^{38}$ T. Schachinger, S. Löffler, M. Stöger-Pollach, and P. Schattschneider, Ultramicroscopy 158, 17-25 (2015). 